OPEN ACCESS

Edited by: Michael Gänzle,

University of Alberta, Canada

Reviewed by:

Guilherme Lanzi Sassaki, Federal University of Paraná, Brazil

Anita Hilda Straus,

Federal University of São Paulo, Brazil

*Correspondence:

Qin Xu

xuqin@gzucm.edu.cn

Qing-Ping Zeng

qpzeng@163.com

Specialty section:

This article was submitted to

Food Microbiology,

a section of the journal

Frontiers in Microbiology

Received: 28 July 2017 Accepted: 22 September 2017

Published: 09 October 2017

Citation:

Wang Q, Huang S-Q, Li C-Q, Xu Q and Zeng Q-P (2017) Akkermansia muciniphila May Determine Chondroitin Sulfate Ameliorating or

Aggravating Osteoarthritis.

Front. Microbiol. 8:1955.

doi: 10.3389/fmicb.2017.01955

\section{Akkermansia muciniphila May Determine Chondroitin Sulfate Ameliorating or Aggravating Osteoarthritis}

\author{
Qi Wang ${ }^{1}$, Shui-Qing Huang ${ }^{2}$, Chang-Qing $L^{3}{ }^{3}$, Qin $X u^{3 *}$ and Qing-Ping Zeng ${ }^{3 *}$ \\ ${ }^{1}$ Clinical Pharmacology Institute, Guangzhou University of Chinese Medicine, Guangzhou, China, ${ }^{2}$ Basic Medical Science \\ College, Guangzhou University of Chinese Medicine, Guangzhou, China, ${ }^{3}$ Tropical Medicine Institute, Guangzhou University \\ of Chinese Medicine, Guangzhou, China
}

Chondroitin sulfate (CS) has shown either ameliorating or aggravating effects on osteoarthritis (OA) in separately conducted clinical trials. Because CS is usually administered orally, it should be affected by or would impact on the individual gut microbiota. Evidence is accumulating that CS can nourish sulfatase-secreting bacteria (SSB) and sulfate-reducing bacteria (SRB). To decipher how can an individual gut microbiota determine the clinical values of CS for treatment on OA, we suggest here that CS would give distinct outcomes for OA treatment depending on Akkermansia muciniphila, a gut commensal probiotic bacterial species as optimal presence albeit also behaving as mucus-eroding bacteria (MEB) when abundant presence. Briefly, CS would ameliorate OA if $A$. muciniphila is present due to without overgrowth of SSB and $\mathrm{SRB}$, whereas CS would aggravate $\mathrm{OA}$ if $A$. muciniphila is absent because of failure in or lack of competition with abundant SSB and SRB. By noting such a frequently ignored phenomenon, we urge the development of non-orally administering CS to minimize its side-effects and extend it to other medicinal applications.

\footnotetext{
Keywords: chondroitin sulfate, osteoarthritis, gut microbiota, probiotic, antibiotic, opportunistic infection, pro-inflammation, anti-inflammation
}

Chondroitin sulfate (CS), also known as polysaccharide constituted of alternated residues of $\mathrm{N}$ acetylgalactosamine and glucuronic acid, is a naturally $\mathrm{O}$-sulfated glycan in cartilage and bones. CS extracted from cow or pig cartilage is prescribed as a symptomatic slow-acting drug for osteoarthritis (OA) in 22 countries, including the European Union, but it is still regulated in the USA as a dietary supplement for improving OA (Jordan, 2003). The United States Food and Drug Administration (FDA) denied a request that CS be labeled as reducing the risk of OA and OA-related joint pain, tenderness, and swelling (FDA, 2004). Despite compelling evidence that CS interferes with OA progression (Vergés and Castañeda-Hernández, 2004; Clegg et al., 2006; Reginster et al., 2007), a systematic review of 20 clinical trials concluded that CS had not demonstrated any symptomatic benefit and discouraged its use in routine clinical practice (Reichenbach et al., 2007). A recent Cochrane clinical trial review concluded that CS appeared to alleviate pain in the short term, but did not improve or maintain the health of joints affected by OA (Singh et al., 2015).

Because of this shortcoming in pain relief, CS was not recommended for the symptomatic treatment of knee OA (Jevseva et al., 2013). Combined treatment with CS and glucosamine sulfate 
or glucosamine hydrochloride did not reduce joint damage in a rabbit model of knee OA (Roman-Blas et al., 2017b). CS plus glucosamine sulfate was not superior to placebo in alleviating articular lesions in a randomized, double-blind, placebo-controlled clinical trial in patients with knee OA (Roman-Blas et al., 2017a).

The effectiveness, benefits, and harms of CS for treating OA are not fully understood, which is possibly attributed to a "good" or "bad" study design. Contamination of heparin with CS has been associated with adverse clinical events, such as allergy and hypertension (Kishimoto et al., 2008), and the potential effect of CS-mediated gut dysbiosis on the differential pharmaceutical outcomes of CS must be considered. Here we aim to give a perspective on the clinical assessment of the impact of CS on OA and other inflammatory disorders.

\section{EXPLAINING INCONSISTENT OUTCOMES OF CS ON OA BY THE INDIVIDUAL-SPECIFICITY OF GUT MICROBIOTA SYMBIONTS}

Considering the oral administration, gastrointestinal uptake, and structural similarity of CS with mucin-type O-glycans of the colonic epithelium, we propose a hypothesis of an Akkermansia muciniphila-dependent CS effect on OA, in which CS improves $\mathrm{OA}$ if A. muciniphila is present, and $\mathrm{CS}$ aggravates $\mathrm{OA}$ if A. muciniphila is absent. A. muciniphila is a species of mucineroding bacteria (MEB) that competes with sulfatase-secreting bacteria (SSB) and the population should increase when SSB are rare and decrease when SSB are abundant. MEB depend on mucin-type $O$-glycans as a sole carbon source; SSB are mucin generalists that can utilize other polysaccharides including those in dietary fiber (Desai et al., 2016).

Colonization of $A$. muciniphila in the gastrointestinal tract varies among individuals (Bressa et al., 2017), and affected by many variables including consumption of fruits and vegetables, drugs, obesity, and surgical history. A. muciniphila has been shown to increase following restriction in dietary fiber and a shift from fiber to mucin consumption (Desai et al., 2016) and increase of Akkermansia spp. has been associated with the antiobesity activity of a polyphenol-rich cranberry extract (Anhê et al., 2015). The antiobesity activity of capsaicin observed in mice fed a high-fat diet was also associated with increase of $A$. muciniphila (Shen et al., 2017). Enrichment of gut $A$. muciniphila populations has been observed in diabetes patients treated with metformin (de la Cuesta-Zuluaga et al., 2017), and following weight loss in obese and/or type 2 diabetes patients (Remely et al., 2016). Roux-en-Y gastric bypass surgery has also been found to increase A. muciniphila populations (Yan et al., 2016).

How can A. muciniphila residing in the gut confer a beneficial effect of CS on OA? Why is CS ineffective or even harmful when SSB preferentially occupy the gut? A. muciniphila may act as a gatekeeper of the mucosa (de Vos, 2017) or stimulate the mucosal immunity to prevent pathogenic invasion, and prompt thickening of the colonic mucosa to reduce the susceptibility to infection. Overgrowth of A. muciniphila is thought to cause mucin degradation, severe damage of the colonic mucosa, extensive endotoxin leakage, and unrecoverable tissue damage. A. muciniphila and B. caccae have been shown to stimulate epithelial access and initiate lethal colitis by the mucosal pathogen Citrobacter rodentium in gnotobiotic mice with a synthetic gut microbiota community (Desai et al., 2016).

Although a small $A$. muciniphila population may induce mucin turnover and a large population may induce colonic mucosal injury, a threshold level has not been identified. If bacterial consumption of mucin-derived nutrients exceeds production, then the integrity of the colonic barrier might be compromised (Rey et al., 2013). The overgrowth of A. muciniphila might derive from genetic immune deficiency. That is supported by the observation that A. muciniphila promoted intestinal inflammation in genetically susceptible germ-free $1110^{-/-}$mice (Seregin et al., 2017).

\section{EVIDENCE FOR THE PROBIOTIC VALUE OF A. MUCINIPHILA}

Evidence of a dose-dependent benefit of A. muciniphila on human health is lacking, but antibiotics capable of eliminating Akkermansia reinforce the colonic mucus barrier and prevent heme-dependent cytotoxic micelles from reaching the epithelium (Ijssennager et al., 2015). A. muciniphila may also be a novel functional microbe with probiotic properties (Dao et al., 2016). Evidence for the therapeutic values of $A$. muciniphila in metabolic diseases includes an inverse correlation with metabolic changes occurring with obesity and type 1 diabetes in mice and humans (Shin et al., 2014; Gomes et al., 2017) and prevention of obesity induced by changes in adipose tissue metabolism and gut barrier function caused by a high-fat diet in mice (Everard et al., 2013).

Favorable effects of $A$. muciniphila on improved glucose tolerance and maintained high interferon (IFN) $\gamma$ levels for controlling various pathogen infections, primarily those caused by intracellular pathogens, have been described by Greer et al. (2016) and Ottman et al. (2017) attributed a positive correlation of A. muciniphila and gut health to immune modulatory effects and immune tolerance to this commensal bacterium. A. muciniphila has been reported to promote intestinal barrier integrity and ameliorate experimental alcoholic liver disease by enhancing colonic mucus thickness and tight-junction expression (Grander et al., 2017). Bifidobacterium that resemble A. muciniphila and degrade mucins by secreting endo- $\alpha-\mathrm{N}-$ acetylgalactosaminidase and 1,2- $\alpha$-L-fucosidase (Ruas-Madiedo et al., 2008) have probiotic behavior that might stimulate mucin overproduction and accelerate replenishment of lost mucins.

The anti-inflammatory activity of CS has been demonstrated in vitro. When CS was immobilized on a scaffold and exposed to proinflammatory cytokines or cytokine-stimulated peripheral blood mononuclear cells, significant increases in the production of molecules with immunosuppressive activity and the expression of their inducible enzymes were observed (Corradetti et al., 2016). Subcutaneous implantation of CS-immobilized scaffold in rats reduced leukocyte infiltration and upregulated genes encoding proteins associated with apoptosis of inflammatory 
cells (Corradetti et al., 2016). CS was found to suppress nuclear NF- $\kappa \mathrm{B}$ translocation in vitro (Tan and Tabata, 2014), and to inhibit NF- $\kappa$ B activation by pathogen- and damage-associated molecules in cultured THP-1 macrophages (Stabler et al., 2017).

\section{PROINFLAMMATORY ACTIVITY OF CS-PROMOTED SSB AND ANTI- INFLAMMATORY ACTIVITY OF ANTIBIOTIC-RESISTANT A. MUCINIPHILA}

CS supports the overgrowth of Bacteroides sp. strain 3452A (an unnamed DNA homology group), B. ovatus, and B. thetaiotaomicron (Lipeski et al., 1986) in the colon and can promote the growth of $B$. thetaiotaomicron, which is an SSB, and Desulfovibrio piger, a species of sulfate-reducing bacteria (SRB) (Rey et al., 2013). SRB may thus thrive if supported by a food chain-like lifestyle, i.e., SRB growth depends on sulfate-containing CS. A number of SSB species, including B. thetaiotaomicron $\mathrm{J} 1$ and 82, B. ovitus E3, and Clostridium hathewayi $\mathrm{R} 4$, have been isolated from healthy subjects (Shang et al., 2016). Theoretically, oral CS administration should support the proportional expansion of SSB and SRB in the distal gut.

We previously reported that daily CS feeding alters the gut microbiota spectrum of mice, but the response varied with individual differences (Figure 1A). In mice with symptoms of early-phase rheumatoid arthritis (RA), Bacillus cereus, an SSB was abundant and $A$. muciniphila was rare compared with normal mice. CS.NM with a normal phenotype has more A. muciniphila, but less $B$. cereus. CS.HL suffering from slight hairless shows no overgrowth of $B$. cereus and A. muciniphila (Liao et al., 2017b). Distinct gut microflora communities might be rapidly established in mice fed CS at identical doses and schedules.

As shown in Figure 1B, when the proliferation of SSB plus $\mathrm{SRB}$ exceeds that of MEB because of unbalanced gut symbionts, $\mathrm{CS}$ would aggravate $\mathrm{OA}$ because of an A. muciniphila population insufficient to stimulate mucin induction and mucus repair. If the gut is kept permanently germ-free, or a commensal diversity of SSB, SRB, MEB, and other symbions is maintained, CS would ameliorate OA (Figure 1C). CS might also ameliorate OA in the presence of normal mucosal immunity and an optimum gut milieu including A. muciniphila. However, CS would aggravate OA given an excess of $A$. muciniphila in a gut with decreased mucosal immunity such as in the immunocompromised, elderly and ill, or patients who are immunosuppressed following organ transplantation (Figure 1D).

We previously reported that CS increased the abundance of Rikenella, a genus of SSB, and Desulfovibrio, a genus of SRB, but did not significantly change the abundance of A. muciniphila. Surprisingly, Rikenella and Desulfovibrio were both eradicated by cephalosporin, but $A$. muciniphila increased. Conversely, Rikenella and Desulfovibrio thrived on exposure to berberine, but $A$. muciniphila was eliminated by this antibiotic analog. Consequently, cephalosporin significantly mitigated colonic mucus lesions and delayed the early pathogenesis of dementia, steatohepatitis, and atherosclerosis. Berberine significantly aggravated colonic mucus lesions and enhanced multi-systemic pathogenesis (Liao et al., 2017a). It has also been observed that germ-free rats had thinner colonic mucus layers, suggesting that microbial colonization is required for mucin production (Desai et al., 2016).

CS has found to upregulate expression of secretory leukocyte protease inhibitor (SLPI), a marker of tissue damage, and mucin 1 (MUC1), a marker of mucus repair; cephalosporin decreased SLPI and MUC1 expression to normal levels (Liao et al., 2017a). The SRB that flourish in association with CS administration could cause a related increase in mucin degradation compared with mucin biosynthesis. In line with the study results, ablation of MUC2 secretion brings bacteria into close contact with the gut epithelium, leading to inflammation and colon cancer (Van der Sluis et al., 2006). The results support the assumption that SSB compete with MEB, and that CS significantly increases the abundance of SSB and restores the A. muciniphila population after SSB are killed by antibiotics.

A pro- and anti-inflammatory activities of CS might reflect the temporal and spatial changes of lipopolysaccharide (LPS) content. We previously reported that peripheral (serum and muscle) LPSs declined to normal levels, and that visceral (hepatic and cardiac tissues) LPSs were elevated to maintain a proinflammatory state, or adipose and cerebral LPSs declined to maintain an anti-inflammatory state (Liao et al., 2017a,b). Administration of of a relative high $1.2 \mathrm{mg} / \mathrm{kg}$ LPS dose led to anti-inflammatory peritoneal changes, and a relative low $0.25 \mathrm{mg} / \mathrm{kg}$ LPS dose caused pro-inflammatory visceral changes, suggesting that anti-LPS treatment reduced LPS in peritoneal but not visceral tissues (Gao et al., 2015). A trend of decrease of anti-inflammatory indicators in the serum following oral CS administration might thus lead to erroneous conclusions of the anti-inflammatory effects of CS.

\section{PERSPECTIVES}

The composition of the gut microbiome influences the pro- and anti-inflammatory activity of CS on aggravation or amelioration of OA via compromise or reinforcement of the colonic mucus barrier. Individual gut microbiota spectra should be carefully considered when evaluating the in vivo effects of CS on OA. The contribution of A.muciniphila in relation to dietary and other etiological variables must be considered to when evaluating the therapeutic values of CS in OA.

Caution should be exercised when administering CS orally to elderly OA patients, who may have decreased mucosal immunity. Similarly, OA patients with a history of organ transplantation and using immunosuppressive drugs might not maintain an $A$. muciniphila population optimal for avoiding persistent colonic mucus erosion and prolonged $\mathrm{OA}$ deterioration.

Development of next-generation CS formulations such as injectable lyophilized CS preparations not only minimizes CSrelated side-effects, but also expands the indication of CS to a broad range of inflammatory disorders including inflammatory bowel disease. Alternatively, it should be an option that CS is used 


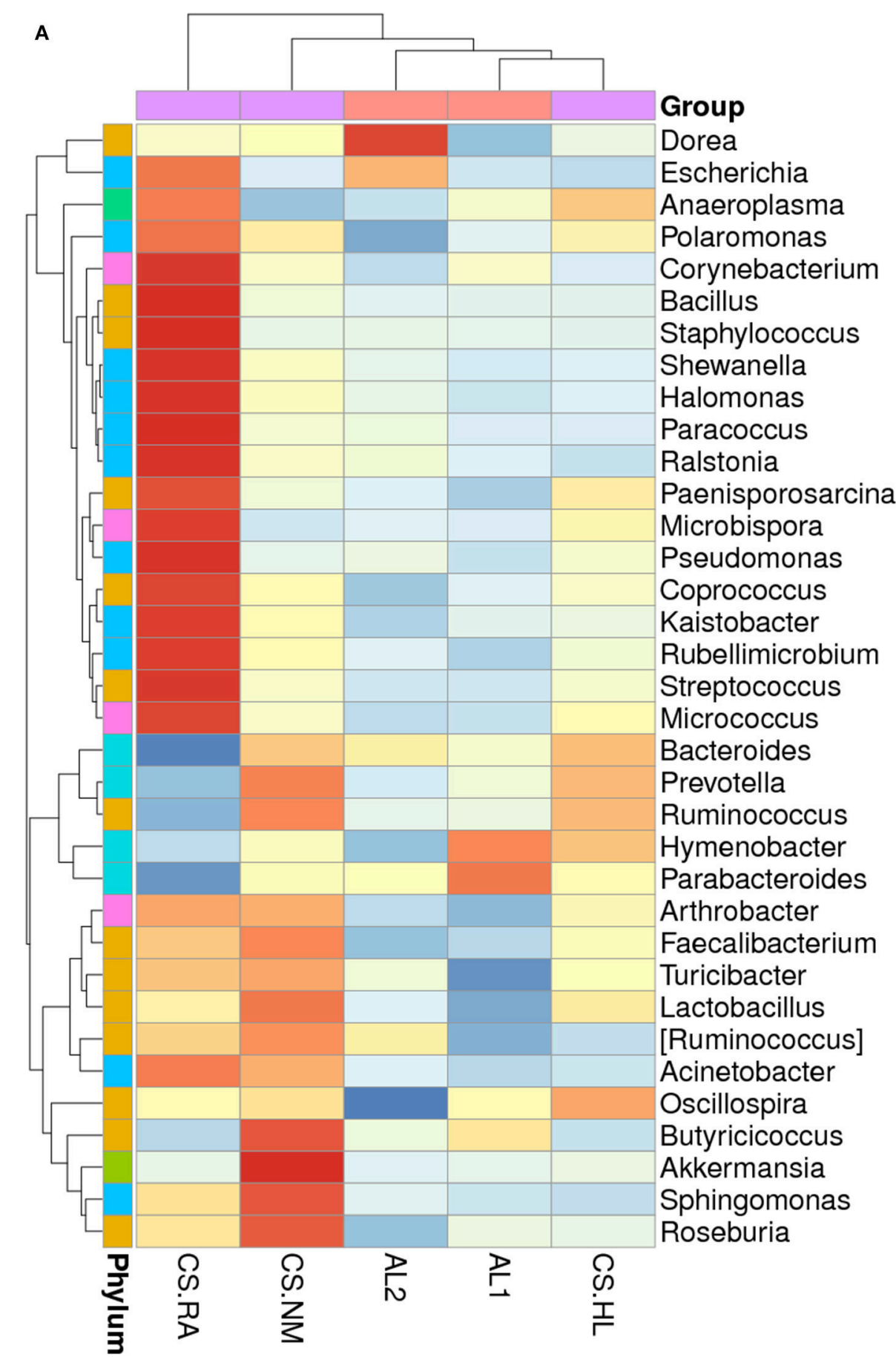

\begin{tabular}{l|l}
\hline 1.5 & Group \\
1 & AL ad libitum \\
0.5 & CS chondroitin \\
0 & \multicolumn{1}{|c}{ sulfate } \\
-0.5 & Phylum \\
-1 & Actinobacteria \\
-1.5 & Bacteroidetes \\
& Firmicutes \\
& Proteobacteria \\
& Tenericutes \\
& Verrucomicrobia
\end{tabular}

\section{OA osteoarthritis}

B Unbalanced gut symbionts

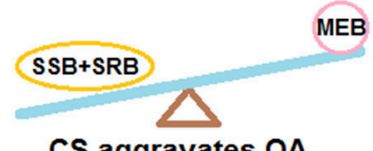

(Insufficient A.muciniphila)

C Balanced gut symbionts

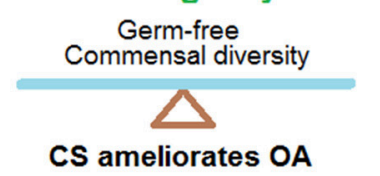

D Unbalanced gut symbionts

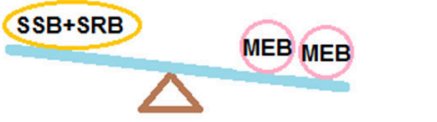

CS ameliorates $O A$ (Optimal A.muciniphila)

\section{CS aggravates OA \\ (Exessive A.muciniphila)}

SSB: sufatase-secreting bacteria SRB: sulfate-reducing bacteria MEB: mucus-eroding bacteria

FIGURE 1 | Changes in the gut microbiota profiles of mice fed ad libitum (AL) and chondroitin sulfate (CS) ( $n=3$ for mice fed CS; $n=2$ for mice fed AL; CS.RA, CS.NM, and CS.HL represent three mice fed CS; AL1 and AL2 represent two mice fed AL). (A) CS-induced gut microbiota shifts that varied in individual mice. (B) Unbalanced gut symbionts with more SSB+SRB than MEB species that aggravate OA due to insufficient $A$. muciniphila; (C) balanced gut symbionts in germ-free conditions or with commensal diversity that ameliorate OA because of no gut microbiota interference. (D) Unbalanced gut symbionts with more MEB than SSB+SRB that ameliorate $\mathrm{OA}$ when the abundance of $A$. muciniphila is optimal or aggravate $\mathrm{OA}$ if the colonization of $A$. muciniphila is excessive. The relative abundance of bacteria is represented by red (increased) to blue (decreased) colors accounting for $Z$ values from -1.5 to 1.5 . 
in combination with probiotics, prebiotics, or emerging dietary intervention (Segarra et al., 2016; Holleran et al., 2017).

\section{AUTHOR CONTRIBUTIONS}

QZ and QW wrote the manuscript. QX, CL, and SH critically reviewed the manuscript. All authors have read and approved the final version of the manuscript.

\section{REFERENCES}

Anhê, F. F., Roy, D., Pilon, G., Dudonné, S., Matamoros, S., Varin, T. V., et al. (2015). A polyphenol-rich cranberry extract protects from diet-induced obesity, insulin resistance and intestinal inflammation in association with increased Akkermansia spp. population in the gut microbiota of mice. Gut 64, 872-883. doi: 10.1136/gutjnl-2014-307142

Bressa, C., Bailén-Andrino, M., Pérez-Santiago, J., González-Soltero, R., Pérez, M., Montalvo-Lominchar, M. G., et al. (2017). Differences in gut microbiota profile between women with active lifestyle and sedentary women. PLOS ONE 12:e0171352. doi: 10.1371/journal.pone.0171352

Clegg, D. O., Reda, D. J., Harris, C. L., Klein, M. A., O’Dell, J. R., Hooper, M. M., et al. (2006). Glucosamine, chondroitin sulfate, and the two in combination for painful knee osteoarthritis. New Eng. J. Med. 354, 795-808. doi: 10.1056/NEJMoa052771

Corradetti, B., Taraballi, F., Minardi, S., Van Eps, J., Cabrera, F., Francis, L. W., et al. (2016). Chondroitin sulfate immobilized on a biomimetic scaffold modulates inflammation while driving chondrogenesis. Stem Cells Transl. Med. 5, 670-682. doi: 10.5966/sctm.2015-0233

Dao, M. C., Everard, A., Aron-Wisnewsky, J., Sokolovska, N., Prifti, E., Verger, E. O., et al. (2016). Akkermansia muciniphila and improved metabolic health during a dietary intervention in obesity: relationship with gut microbiome richness and ecology. Gut 65, 426-436. doi: 10.1136/gutjnl-2014-3 08778

de la Cuesta-Zuluaga, J., Mueller, N. T., Corrales-Agudelo, V., Velásquez-Mejía, E. P., Carmona, J. A., Abad, J. M., et al. (2017). Metformin is associated with higher relative abundance of mucin-degrading Akkermansia muciniphila and several short-chain fatty acid-producing microbiota in the gut. Diabetes Care 40, 54-62. doi: 10.2337/dc16-1324

de Vos, W. M. (2017). Akkermansia muciniphila: a conserved intestinal symbiont that acts as the gatekeeper of our mucosa. Microbiology 163, 646-648. doi: $10.1099 /$ mic. 0.000444

Desai, M. S., Seekatz, A. M., Koropatkin, N. M., Kamada, N., Hickey, C. A., Wolter, M., et al. (2016). A dietary fiber-deprived gut microbiota degrades the colonic mucus barrier and enhances pathogen susceptibility. Cell 167, 1339, e21-1353. e21. doi: $10.1016 /$ j.cell.2016.10.043

Everard, A., Belzer, C., Geurts, L., Ouwerkerk, J. P., Druart, C., Bindels, L. B., et al. (2013). Cross-talk between Akkermansia muciniphila and intestinal epithelium controls diet-induced obesity. Proc. Natl. Acad. Sci. U.S.A. 110, 9066-9071. doi: 10.1073/pnas.1219451110

FDA (2004). Letter Regarding the Relationship Between the Consumption of Glucosamine and/or Chondroitin Sulfate and a Reduced Risk of: Osteoarthritis; Osteoarthritis-Related Joint Pain, Joint Tenderness, and Joint Swelling; Joint Degeneration; and Cartilage Deterioration (Docket No. 2004P-0059). Addressed to John W. Emford, Esq. William K. Hubbard. Available online at: http://www. fda.gov/food/ingredientspackaginglabeling/labelingnutrition/ucm073400.htm

Gao, Q., He, J., Liao, T., and Zeng, Q. P. (2015). 2,4-dinitrophenol downregulates genes for diabetes and fatty liver in obese mice. J. Biosci. Med. 3, 44-51. doi: $10.4236 / j b m .2015 .39007$

Gomes, J. M., Costa, J. A., and Alfenas, R. C. (2017). Metabolic endotoxemia and diabetes mellitus: a systematic review. Metab. Clin. Exp. 68, 133-144. doi: 10.1016/j.metabol.2016.12.009

Grander, C., Adolph, T. E., Wieser, V., Lowe, P., Wrzosek, L., Gyongyosi, B., et al. (2017). Recovery of ethanol-induced Akkermansia muciniphila depletion ameliorates alcoholic liver disease. Gut. doi: 10.1136/gutjnl-2016-313432. [Epub ahead of print].

\section{ACKNOWLEDGMENTS}

We thank Jiang He, Tao Liao, and Yan-Ping Chen, and Li-Li Tan for their assistance in manuscript preparation. This work was supported by the National Natural Science Foundation of China (NSFC, No. 81273817 and No. 81473740 to QW; No. 81673861 to $\mathrm{CL}$ ) and Guangdong Science and Technology Plan Project (No. 20150404042 to QX).

Greer, R. L., Dong, X., Moraes, A. C. F., Ryszard, A., Zielke, R. A., Fernandes, G. R., et al. (2016). Akkermansia muciniphila mediates negative effects of IFN $\gamma$ on glucose metabolism. Nat. Commun. 7:13329. doi: 10.1038/ncomms13329

Holleran, G., Lopetuso, L. R., Ianiro, G., Pecere, S., Pizzoferrato, M., Petito, V., et al. (2017). Gut microbiota and inflammatory bowel disease: an update. Minerva. Gastroenterol. Dietol. 63, 373-384. doi: 10.23736/S1121-421X.17.02386-8

Ijssennager, N., Belzer, C., Hooiveld, G. J., Dekker, J., van Mil, S., and W., Müller, M., et al. (2015). Gut microbiota facilitates dietary heme-induced epithelial hyper-proliferation by opening the mucus barrier in colon. Proc. Natl. Acad. Sci. U.S.A. 112, 10038-10043. doi: 10.1073/pnas.1507645112

Jevseva, D. S., Brown, G. A., Jones, D. L., Matzkin, E. G., Manner, P. A., Mooar, P., et al. (2013). The American Academy of orthopaediac surgeons evidence-based guideline on: treatment of osteoarthritis of the knee, 2 nd edition. J. Bone Joint Surg. Am. 95, 1885-1886. doi: 10.5435/JAAOS-21-09-571

Jordan, K. M. (2003). An evidence based approach to the management of knee osteoarthritis: report of a task force of the standing Committee for International Clinical Studies Including Therapeutic Trials (ESCISIT). Ann. Rheum. Dis. 62, 1145-1155. doi: 10.1136/ard.2003.011742

Kishimoto, K., Viswanathan, K., Ganguly, T., Elankumaran, S., Smith, S., Pelzer, K., et al. (2008). Contaminated heparin associated with adverse clinical events and activation of the contact system. New Engl. J. Med. 358, 2457-2467. doi: 10.1056/NEJMoa0803200

Liao, T., Chen, Y. P., Huang, S. Q., Tan, L. L., Li, C. Q., Huang, X. A., et al. (2017a). Chondroitin sulfate elicits systemic pathogenesis in mice by interfering with gut microbiota homeostasis. BioRxiv doi: 10.1101/142588

Liao, T., Chen, Y. P., Tan, L. L., Li, C. Q., Wang, Q., Huang, S. Q., et al. (2017b). Chondroitin sulfate flourishes gut sulfatase-secreting bacteria to damage mucus layers, leak bacterial debris, and trigger inflammatory lesions in mice. BioRxiv doi: 10.1101/145714

Lipeski, L., Guthrie, E. P., O’Brien, M., Kotarski, S. F., and Salyers, A. A. (1986). Comparison of proteins involved in chondroitin sulfate utilization by three colonic Bacteroides species. Appl. Environ. Microbiol. 51, 978-984.

Ottman, N., Reunanen, J., Meijerink, M., Pietilä, T. E., Kainulainen, V., Klievink, J., et al. (2017). Pili-like proteins of Akkermansia muciniphila modulate host immune responses and gut barrier function. PLOS ONE 12:e0173004 doi: 10.1371/journal.pone.0173004

Reginster, J. Y., Heraud, F., Zegels, B., and Bruyere, O. (2007). Symptom and structure modifying properties of chondroitin sulfate in osteoarthritis. Mini Rev. Med. Chem. 7, 1051-1061. doi: 10.2174/138955707782110088

Reichenbach, S., Sterchi, R., Scherer, M., Trelle, S., Bürgi, E., Bürgi, U., et al. (2007). Meta-analysis: chondroitin for osteoarthritis of the knee or hip. Ann. Intern. Med. 146, 580-590. doi: 10.7326/0003-4819-146-8-200704170-00009

Remely, M., Hippe, B., Zanner, J., Aumueller, E., Brath, H., and Haslberger, A. G. (2016). Gut microbiota of obese, type 2 diabetic individuals is enriched in Faecalibacterium prausnitzii, Akkermansia muciniphila and Peptostreptococcus anaerobius after weight loss. Endocr. Metab. Immune Disord. Drug. Targets 17, 99-106. doi: 10.2174/1871530316666160831093813

Rey, F. E., Gonzalez, M. D., Cheng, J., Ahern, P. P., and Gordon, J. I. (2013). Metabolic niche of a prominent sulfate-reducing human gut bacterium. Proc. Natl. Acad. Sci. U.S.A. 110, 13582-13587. doi: 10.1073/pnas.1312524110

Roman-Blas, J. A., Castaneda, S., Sanchez-Pernaute, O., Largo, R., HerreroBeaumont, G., et al. (2017a). Combined treatment with chondroitin sulfate and glucosamine sulfate shows no superiority over placebo for reduction of joint pain and functional impairment in patients with knee osteoarthritis: a six-month multicenter, randomized, double-bland, placebo-controlled clinical trial. Arthritis Rheumatol. 69, 77-85. doi: 10.1002/art.39819 
Roman-Blas, J. A., Mediero, A., Tardio, L., Portal-Nunez, S., Gratal, P., HerreroBeaumont, G., et al. (2017b). The combined therapy with chondroitin sulfate plus glucosamine sulfate or chondroitin sulfate plus glucosamine hydrochloride does not improve joint damage in an experimental model of knee osteoarthritis in rabbits. Eur. J. Pharmacol. 794, 8-14. doi: 10.1016/j.ejphar.2016.11.015

Ruas-Madiedo, P., Gueimonde, M., Fernández-Garcia, M., de los Reyes-Gavilán, C. G., and Margolles, A. (2008). Mucin degradation by Bifidobacterium strains isolated from the human intestinal microbiota. App. Environ. Microbiol. 74, 1936-1940. doi: 10.1128/AEM.02509-07

Segarra, S., Martinez-Subiela, S., Cerda-Cuellar, M., Martinez-Puig, D., Munoz-Prieto, A., Rodriguez-Franco, F., et al. (2016). Oral chondroitin sulfate and probiotics for the treatment of cannie inflammatory bowel disease: a randomized, controlled clinical trial. BMC Vet. Res. 12:49. doi: 10.1186/s12917-016-0676-x

Seregin, S. S., Golovchenko, N., Schaf, B., Chen, J., Pudlo, N. A., Mitchell, J., et al. (2017). NLRP6 protects Il10-/- mice from colitis by limiting colonization of Akkermansia muciniphila. Cell Rep. 19:2174. doi: 10.1016/j.celrep.2017.05.074

Shang, Q., Yin, Y., Zhu, L., Li, G., Yu, G., and Wang, X. (2016). Degradation of chondroitin sulfate by the gut microbiota of Chinese individuals. Int. J. Biol. Micromol. 86, 112-118. doi: 10.1016/j.ijbiomac.2016.01.055

Shen, W., Shen, M., Zhao, X., Zhu, H., Yang, Y., Lu, S., et al. (2017). Anti-obesity effect of capsaicin in mice fed with high-fat diet is associated with an increase in population of the gut bacterium Akkermansia muciniphila. Front. Microbiol. 8:272. doi: 10.3389/fmicb.2017.00272

Shin, N. R., Lee, J. C., Lee, H. Y., Kim, M. S., Whon, T. W., Lee, M. S., et al. (2014). An increase in the Akkermansia spp. population induced by metformin treatment improves glucose homeostasis in diet-induced obese mice. Gut 63, 727-735. doi: 10.1136/gutjnl-2012-303839

Singh, J. A., Noorbaloochi, S., MacDonald, R., and Maxwell, L. J. (2015). Chondroitin for osteoarthritis. Cochrane Data. Sys. Rev. 1:CD005614. doi: 10.1002/14651858.CD005614.pub2
Stabler, T. V., Huang, Z., Montell, E., Vergés, J., and Kraus, V. B. (2017) Chondroitin sulphate inhibits NF- $\mathrm{BB}$ activity induced by interaction of pathogenic and damage associated molecules. Osteoarthr. Cartil. 25, 166-174. doi: 10.1016/j.joca.2016.08.012

Tan, G. K., and Tabata, Y. (2014). Chondroitin-6-sulfate attenuates inflammatory responses in murine macrophages via suppression of NF- $\mathrm{B}$ nuclear translocation. Acta Biomater. 10, 2684-2692. doi: 10.1016/j.actbio.2014. 02.025

Van der Sluis, M., De Koning, B. A., De Bruijn, A. C., Velcich, A., Meijerink, J. P., Van Goudoever, J. B., et al. (2006). Muc2-deficient mice spontaneously develop colitis, indicating that MUC2 is critical for colonic protection. Gastroenterology 131, 117-129. doi: 10.1053/j.gastro.2006.04.020

Vergés, J., and Castañeda-Hernández, G. (2004). On the bioavailability of oral chondroitin sulfate formulations: proposed criteria for bioequivalence studies. Proc. West. Pharmacol. Soc. 47, 50-53.

Yan, M., Song, M. M., Bai, R. X., Cheng, S., and Yan, W. M. (2016). Effect of Roux-en-Y gastric bypass surgery on intestinal Akkermansia muciniphila. World J. Gastrointest. Surg. 8, 301-307. doi: 10.4240/wjgs.v8. i4.301

Conflict of Interest Statement: The authors declare that the research was conducted in the absence of any commercial or financial relationships that could be construed as a potential conflict of interest.

Copyright (C) 2017 Wang, Huang, Li, Xu and Zeng. This is an open-access article distributed under the terms of the Creative Commons Attribution License (CC $B Y)$. The use, distribution or reproduction in other forums is permitted, provided the original author(s) or licensor are credited and that the original publication in this journal is cited, in accordance with accepted academic practice. No use, distribution or reproduction is permitted which does not comply with these terms. 